\title{
Cognition, Human Evolution and the possibilities for an Ethics of Warfare and Peace
}

Accepted for publication in Social History \& Evolution) (https://www.sociostudies.org/journal/seh/forthcoming/)

\author{
Daniel Barreiros \\ Associate Professor, Institute of Economics, Federal University of Rio de Janeiro, \\ UFRJ, Brazil. Researcher at the Bioethics and Applied Ethics Center (UFRJ, Brazil). \\ daniel.barreiros@ie.ufrj.br \\ Daniel Ribera Vainfas \\ $\mathrm{PhD}$ student, Institute of Economics, Federal University of Rio de Janeiro, \\ UFRJ,Brazil. Researcher at the Bioethics and Applied Ethics Center (UFRJ, Brazil). \\ danielvainfas@gmail.com
}




\section{Abstract}

African apes live in large stable social groups with an increased potential for conflict between individual agendas; a reasonable volume of evidence has suggested an instinctive and ethological basis for their intrasocial ethical behavior. Would there be some innate and ethological behavioral basis for ethics in human intersocietal relations, whether in war (providing the limits to the exercise of hard power), or in peace (establishing standards for preservation of intersocietal non-hostility)? As a hypothesis, we suggest that human exclusivity in the exercise of the ethics of warfare and peace is a product of the human transdominial cognition, capable of recombining and re-signifying innate behavioral algorithms through culture, applying them to absolutely innovative functions. 


\section{Ethics as behavior, ethics as reflection}

Ethics, as a field of philosophy itself, unfolds into incalculable ramifications. Despite this, it is possible to identify two major orientations that guide the debates on ethics. On the one hand, there are those who understand ethics as a spontaneous manifestation of certain behavior (e.g. altruism, cooperation, rewards and punishments). On the other hand, ethics appears as a phenomenon that requires self-reflection, so an action can only be considered truly ethical when the agent responsible for it is aware of the act it performs. These orientations are rooted in the moral philosophy of two eighteenth-century authors: on the one hand, Hume, in arguing that human passions are the moral compass, is the exponent of the first view; in opposition to it, Kant, in defending the rational position before reality, represents the second orientation. For the purpose of this work, we are interested in a more specific question: could the ethical behavior of nonhuman animals shed some light on the debate on ethics among humans?

It is known from the biological literature that social animals (especially mammals), a group that includes $H$. sapiens, possess a series of autonomous mechanisms that regulate social relations, determining the instances of conflict and cooperation. The general problem of animal ethics can be divided into two major paradigms: the exclusive and the inclusive, whose fundamental distinction is the incorporation or non-incorporation of nonhuman animals into what is considered ethical. Considering the philosophy of biology, there is a consensus that ethics derives from natural selection, constituting itself from a biological substrate, that forms the basis for all possible intercultural variations (Ruse 2010; Ayala 2010). In this sense, ethical codes, like all cultural manifestations, exist in constant dialogue with biology, thus being a culturally specific manifestation of a fundamental element rooted in human constitution (Tooby and Cosmides 2015: 7).

The great divergence is set between what constitutes the general substratum and what constitutes the variant. In some way, the problem is the same formulated by Hume about human thought. When we think about reality, we begin with a general and relatively certain element about which there is general agreement, then 
we move into unknown territory where perception is less precise and debates are more intense. The central element upon which scientific consensus exists lies in natural selection and the innate capacity for ethics; the nebulous area is represented by the constitution of the ethical norms.

In contemporary debate, we can distinguish three distinct levels of interpretation about animal ethics. These levels correspond to a gradation of maximal, mean, and minimal agreement on the inclusion of nonhuman animals in the condition of ethical creatures (Fitzpatrick 2017). Those who do not include these animals under any circumstances as ethical creatures are the ones who identify that ethics is constituted not by ethical behavior itself but by critical reflection on behavior. Thus only beings endowed with a sufficient degree of rationality would be considered ethical. On this, Ayala (2010: 326) says:

The capacity for ethics is an outcome of gradual evolution, but is an attribute that only exists when the underlying attributes (i.e., the intellectual capacities) reach an advanced degree. The necessary conditions for ethical behavior only come about after the crossing of an evolutionary threshold. The approach is gradual, but the conditions only appear when a degree of intelligence is reached such that formation of abstract concepts and the anticipation of the future are possible, even though we may not be able to determine when the threshold was crossed

On the opposite side, there are those who argue that ethics is defined by ethical behavior itself; so, as social animals exhibit ethical behaviors (e.g., rewarding certain behaviors and punishing others, exhibiting altruistic attitudes toward members of the group) they would be unequivocally ethical creatures. Whether or not they think about their own actions would be somewhat devoid of consequence (Fitzpatrick 2017; Ruse 2010):

(...) we humans have built-in innately, or instinctively if you like, a capacity for working together socially. And this capacity manifests itself at the physical level as a moral sense. Hence morality or, rather, a moral sense is something which is hard-wired into humans - mediated and fashioned by culture. 
Morality has been put there by natural selection in order to get us to work together socially or to cooperate (Ruse 2010: 307).

Between the poles, there are those who postulate ethical animal behavior as a step toward human ethics; in that sense, the difference between nonhuman animals and human animals would be a matter of intensity and not of quality. Here the idea of a gradualist ethic is framed by the image of De Waal's tower (2006: 181):

To neglect the common ground with other primates, and to deny the evolutionary roots of human morality, would be like arriving at the top of a tower to declare that the rest of the building is irrelevant, that the precious concept of 'tower' ought to be reserved for its summit. While making for good academic fights, semantics are mostly a waste of time. Are animals moral? Let us simply conclude that they occupy several floors of the tower of morality. Rejection of even this modest proposal can only result in an impoverished view of the structure as a whole.

If we return to Ayala (2010), in defense of an exclusive paradigm of ethics, we will see that the author presents it as an exaptation, rather than seeing it as an adaptation. Exaptation is a phenomenon of natural selection in which a particular behavior or a given anatomical structure is not directly selected to perform the function associated with it. An exaptation occurs when there is a change in the use of a structure (physical or behavioral) without a change in the structure itself. Fundamentally, this means that, even if ethics is a concrete biological reality, it was not the direct result of natural selection; that is, the basis for ethical behavior, was selected, not ethics itself.

In particular, Ayala (2010) identifies three general fundamentals for the phenomenon of ethics:

1- the ability to predict the outcome of an action;

2- the ability to judge the outcome of an action;

3- the ability to choose whether or not to perform an action. 
There are interesting issues to be raised against Ayala's formulation (2010). At the core, his argument is that ethics exists only when these three statements are simultaneously true, and only when an organism has a high degree of rationality. In the end, it would mean that ethics is a human exclusivity, arising from rational thought. As for the first statement, it is relatively simple to recognize that it is not exclusive to human beings: the ability to relate means and ends is relatively common among mammals, and even though there is abstract thinking among humans, it is quite possible to recognize that some animals are able to predict the consequences of their actions. The second statement is more interesting. Even though social animals are capable of rewarding and punishing certain general behaviors, postulating that they do so based on a judgment of values can be excessive. Thus, the second statement takes us back to the initial position: we are faced with a situation in which ethics is defined as a product of the critical reflection of the individual and, therefore, beyond experimental observation (up to this moment).

Finally, we have the most interesting point in the third statement. Fundamentally, Ayala's (2010) argument is that ethics needs free will to exist; if there is no free will, then there is no room for reflection. In this context, the author approaches Machiavelli, who, in admitting his inability to state whether life is dominated by Fortuna or Virtù, chooses to delegate $50 \%$ of everything to each one, because he recognizes that no sort of political science would be possible without the human capability of interference on the future, to a certain extent.

Despite this elusive response, there is an empirical challenge to Ayala's third claim. There is a relevant body of studies in neuroscience (Libet 1985; Soon et al. 2008; Bode et al. 2011) that supports that, in some cases, human ability to make a decision is an elaborate process of rationalization of a choice that has already been made by the brain, moments before. Thus, by extrapolation, at least a fraction of what we consider free will would be an illusion.

Of course, the existence or the absence of free will is one of longest philosophical debates in human history, so that it is beyond the scope of this work to solve it. However, we can propose a counterfactual challenge to Ayala's (2010) 
thesis: if there is no free will, in fact would there be no ethics? Would there be a formulation of the concept of ethics that could dismiss the notion of freedom?

Curiously, Ruse (2010) in direct debate with Ayala offers us an answer to this consideration. Ruse proposes that ethics is, actually, a biologically programmed lie whose existence is justified by its effectiveness. It is aimed at the ultimate biological purpose, the maintenance of life. Essentially, Ruse proposes an ethics of feelings, that is, if a particular attitude is felt by the subject as wrong, that attitude should be unethical. Since feelings are both biologically conditioned and relatively general, then ethics is a relatively autonomous and functional biological construct, as an anatomical part; in this sense, ethics could prescind from free will.

So in spite of the differences, the approaches are more similar than they appear. Fundamentally, the point of disagreement is a matter of definition, about whether or not ethics needs reflection to exist. The existence of ethical behavior among social animals is not questioned, particularly among mammals. Thus, an approach to the consequences of ethics can abridge the idiosyncrasies of the authors, and may consider that the existence of ethical behavior is sufficient for the most important conclusions. More specifically, to the problem of warfare, it does not matter whether true altruism (or its absence, depending on whether we take in consideration the 'enemy' or the 'allies') requires critical reflection on the part of the agent; since there is a natural altruism common to social primates, then, behavior must manifest itself despite rational considerations. Nevertheless, the same cannot be said of an ethics of warfare and peace, as we shall see.

\section{Ethics as Behavior and the Archeology of Mind}

From the points of view of the archeology of the mind and of the evolutionary psychology, the controversy over the dyad 'ethical behavior - rational ethics' can be sufficiently accommodated, so that aspects that at first glance appear to be exclusive may form an image of simultaneity or even feedback. We believe we can work - in dialectical terms - both with the presence of ethics in the form of ethology (i.e., innate behavior) and in the form of cultural products. This game of scales is in the core of the debates on the relation between intrasocietal ethics, intersocietal conflict 
and the evolutionary possibilities for an ethics of warfare and peace in the long duration of the Big History. From a primatological angle, we see that great social apes, particularly chimpanzees (Pan troglodytes, the closest extant evolutionary cousin of $H$. sapiens), are ethologically endowed with specialized cognitive mechanisms for mediating intra-group conflicts, mechanisms that provide some sort of 'unstable order' in the management of disputes between individual agendas; by reducing the degree of agonistic behavior inside the social unit, and especially the intensity of interpersonal lethal violence (conditions for the permanent sociability), this ethological constraints are an important asset in the struggle for the collective expansion of reproductive fitness. Whether manifested by patterns of behavior with hardwired contents, or by innate-directed attention to culturally agreed ethical norms, this general aspect is common to two long evolutionary lineages, which diverged from a common ancestor about six million years in the past, and have resulted in modern humans and common chimpanzees. At the same time, we are familiar with the unbounded violence characteristic of coalitional conflict between different social groups of common chimpanzees, in which there are no ethological constraints to lethality, nor innate mechanisms that determine when and how the 'state of war' is suspended, albeit provisionally. In the same vein, from the battlefields of Jebel Sahaba and Talheim, prior to the Bronze Age (Keeley 1996), through the inter-state systems of Antiquity to the present day, the history of human warfare suggests the great frequency with which unrestricted violence can take control of intersocietal relations. In simple terms, 'War is Hell' summarized W. Tecumseh Sherman to the graduates of the Michigan Military Academy in 1879.

Thus, some version of an intra-group social ethics, emerging innately among humans, chimpanzees and other social primates, assumes different degrees of complexity according to each species, to the ecological conditions in which they evolved, to the niches that they occupy, to the size of their social units and to their phylogenetic history. In all these cases, ethics is the product of a social intelligence with different degrees of complexity, of neurocognitive specialization, and of integration with general intelligence, as well as with other mental modules. This social intelligence, which may be composed of disconnected and dedicated mental modules, or of a high-energy cognitive domain, composed of these modules, is the source of the ethology of conflict resolution among social primates; in this sense, 
'ethics' emerges as understood by Fitzpatrick and Ruse, who emphasize the innate and behavioral dimension of the phenomenon (common to many primates), minimizing the human dimension of it (the entanglement between ethology and culture, as in $\mathrm{H}$. sapiens). As part of this general scenario, humans are ethologically compelled to conceive their social relations with other humans in ethical terms, whose specific contents turn out to be culturally shaped, and based on a body of more or less 'universal themes'. Humans do not have immediate and automatic access to all the contents that inhabit their collective unconscious (that harbors all the ethological complexes in the metarepresentational mind), but in their social relations they are innately attentive to information from the environment that echoes moral and ethical issues in the unconscious mind (distributive criteria, recognition of prestige, 'justice', etc.) and that end up invoking archetypal images and responses, full of cultural contents, but rooted in the deep psyche. On the other hand, we are not innately aware of information regarding fluctuations in the Earth's magnetic field, as many species of migratory birds are; the regulation of intrasocial conflict is as vital for primates as it is determinant for certain species of birds to find their way to meridional places when the northern winter is coming. Thus, this power to resort to ethology, to a lesser extent in its predetermined contents and values, and to a greater in the condition of a behavioral algorithm on which cultural values are applied, is a function of the transdominial and metarepresentational mind, an evolutionary innovation absolutely unprecedented and recent in the natural history of primates, which, bringing a greater degree of complexity to the problem of ethics, converges with de Waal's gradualist conception.

\section{Ethics of warfare and peace: between ethology and culture}

But what about an ethics of warfare and peace? Is it a ubiquitous primatological possibility? In this case, only humans seem capable of establishing some form of ordering in their intergroup relations, as well as norms on the limits of war; and in no way does this seem to come from an ethological predisposition to intersocietal ethics. Like common chimpanzees, we humans are not ethologically bound to restraints and normativity in our interactions with conspecifics that are members of other social groups. Therefore, humans become capable of unrestricted violence against anyone interpreted as the 'other', when cultural and institutional 
restraints are shaken for some reason. It follows that, in pursuing a path convergent to that proposed by Ayala, humans emerge as incapable of being 'ethical' exclusively in ethological terms in their intersocietal relations (in fact, they are incapable of being exclusively ethological in any behavioral domain); this implies that, inevitably, ethics among humans involves the capacity for reflection and, to some extent, for making behavioral choices. This is a typical attribute of the metarepresentational and transdominial mind, so that we are able to look as an external observer to our behavioral algorithms (our human ethology, so to say). In this process, humans have a slight opportunity to scrutinize these ethological contents, rules and expectations, with varying degrees of success; by doing so, they can opt in some way for the application of these innate contents, rules and expectations to certain situations, deliberate about the terms of that same application, and about the situation in which they will be applied. Thus, it is this 'rational' dimension of ethics emphasized by Ayala that empowers us to apply algorithms dedicated to regulating our intra-group relations to contexts in which the counterpart of the social relation is not recognized as 'one of us.' Among common chimpanzees, any conspecific outside the hierarchical pyramid of intra-group relations is not object of the ethology of conflict regulation ('ethics' according to Fitzpatrick and Ruse) and therefore can be a target of lethal violence if the circumstances allow for it. Among humans, our natural ability to 'confuse' ethological contents, and apply them to situations other than those for which they evolved and were adaptive (and that is why Ayala understands 'ethics' as exaptation) enables us to look at the 'other,' at the 'foreign', as capable of being integrated into our innate mechanisms for resolving conflicts. The same cognitive vicissitude that leads us to talk to pets (despite knowing 'rationally' that they do not fully understand our conceptual language), or to treat them as subject of rights (as bioethics activists and citizens do in a daily basis) allows us to 'raise the white flag', 'bury the hatchet', declare a 'just' war or sign the Geneva Convention.

\section{Selective pressures for ethics as behavior}

Let us return to social, intra-group ethics. In the lineage leading to $P$. troglodytes and $H$. sapiens, what would have been the selective pressure that made the ethological instruments of conflict management become so essential? Of course, other species of social primates, unrelated to the common ancestor we share with 
chimpanzees, also developed innate rules for the management of individual agendas with no damage to permanent sociability, but which, with distinct evolutionary histories, may have been driven by challenges and phenomena specific and different from those we believe were behind the adaptation of the last common ancestor between humans and chimpanzees to their environment. In this way, and avoiding extrapolations, let us concentrate on our common ancestor. Let us recognize that, through a parsimonious and phylogenetic interpretation, a social aspect potentially present in its behavior may have been crucial to the ethology of 'intrasocial ordering' in humans and chimpanzees: patrilineal, patrilocal, masculine and cooperative sociability. Permanent social cooperation among males is a phenomenon quite rare in the natural history of primates. It is much more common that related females form the core of primitive social groups, along with their daughters (nulliparous or sexually mature) and their sons (before sexual maturity) (Fleagle 2013: 45). Kin cooperation among females is a long-lasting behavioral trait whose emergence preceded in many millions of years the permanent male sociability. In species with this social pattern, males form unstable and uncooperative groups on the periphery of the female vital space, competing with each other for sexual opportunities, and periodically migrating to other social units.

In certain cases, harems are formed in which a single dominant male seeks to exert sexual exclusivity over cooperative kin-related females, excluding, as far as possible, other males from access to mating opportunities through intense agonistic behavior, involving a high degree of physical violence. This is not the pattern of sociability suggested for the last common ancestor between humans and chimpanzees; in it, the matrix of cooperation involves not only females (non-kin related, since they migrate to other groups upon reaching sexual maturity), but especially kin-related males. In a context in which the perceptiveness of male dominance locus is diluted, as happens among humans and common chimpanzees, the absence of clear dominance paves the way to the conflict between individual masculine agendas; sooner or later, this conflict would easily escalate towards a fratricidal struggle involving kin-related males. To cope with this, a set of innate behaviors were naturally selected, which, by preserving the patrilineal masculine groups, make it so through hierarchies with clear ritualistic dimension with mutual recognition of the status of kin-related peers. Hierarchy recognition reduces intra- 
group agonistic behavior (i.e. between related males) and lethal violence by setting 'escape clauses' that allow an individual to challenge the position of a particular peer, putting in motion a circulation mechanism applied to the ranks of dominance. Similarly, social relations between females also conform to hierarchical patterns, although less intense and ritualized than among males (Foley 2008; Wrangham and Peterson 1996).

The establishment of fluid hierarchies, with vertical movements and countermovements and status-gaining strategies, was central to the maintenance of permanent social groups, with kin-related male cooperation. The complexity of societies with a strong occurrence of fusion-fission processes, as happens with humans and common chimpanzees (Fleagle 2013: 45-46; Aureli et al. 2008), could hardly be managed by the use of general intelligence, an instrument that is present in varying degrees of development in many species of primates (especially anthropoids). By general intelligence we designate a set of multi-use cognitive instruments, whose main characteristic lies in functioning as an alternative circuit to simple ethological modular contents. General intelligence manifests itself in parallel with these ethological modules, without eliminating them; it seizes and processes sensorial information, in order to learn from the interaction with the environment. This interaction, however, is mediated by generic rules, applicable to any cognitive contexts and challenges, based on trial and error (tuning behavior according to the results of actions and processes). Unstable forms of sociability, with frequent partner changes, and greater possibility of conflict resolution through a simple 'fight or flight' dynamics, can be sufficiently managed through the operation of a few innate mental modules dedicated to sociability, and by the fine tuning given by the generic instruments of general intelligence.

\section{Specialized social intelligence and ethics as behavior}

The challenges of permanent sociability, especially in evolutionary contexts when the perception of male dominance locus is blurred (post-harem societies), seem to go hand in hand with the evolution of a cognitive domain specialized in conflict management, with rules and mechanisms of attention specifically focused on this goal. If general intelligence focuses on event contingencies, through generic 
tools applicable to any other interactions with the environment, certainly it was not enough to deal with the complexity of the social life among humans and common chimpanzees, and their direct ancestors; in this case, the development of a social intelligence composed of a specialized and highly efficient network of ethological modules (isolated from the general intelligence) resulted in the following competences: 1) analyzing carefully all kinds of sensory information that signals the current status rank of a conspecific; 2) analyzing information that allows mapping social relations between two or more individuals, with triangulations that may include or not the observer; 3 ) formulating hypotheses about the possibilities of rise and fall of individuals or coalitions in the rank stratification, and of developing strategies of personal repositioning in the hierarchical pyramid (Mithen 2002; Wrangham and Peterson 1996). Specialized social intelligence employs the observer's own mind as a model to test hypotheses about the behavior of third parties; through welldeveloped empathic abilities, the observer hypothetically puts himself in the analyzed situation and ponders, based on his own simulated reactions, upon the most likely courses of action that would be taken by a counterpart in a given context. This analysis is, in a second moment, modulated by information about the circumstances of the action and about the temperament of the analyzed individual (i.e., matching the ability to predict the outcome of an action, the first condition for the social ethics established by Ayala).

\section{Rite, hierarchy, and prosocial brakes}

The ethology of the struggle for status among common chimpanzees is regulated precisely by these mechanisms of social intelligence, which makes hierarchical relations to function without the excesses of interpersonal lethal violence (although deviations from this norm certainly occur, due to particular reasons). The specialization of social cognition, generating ethological norms of behavior in the interaction between conspecifics ('ethics' according to Fitzpatrick and Ruse) also produces, in chimpanzees, awareness of the self and of others; but, isolated from the general intelligence, that cognitive social domain produces only consciousness of the self and the others as social actors, and only in the very exercise of social relations. It happens to be, then, a kind of consciousness focused on a specific task, with strong ethological algorithms and contents, inapplicable to any other contexts of 
interaction with the environment, since it lacks full and fluid articulation with the rest of the primate psyche. It is absolutely not an 'awareness of consciousness' that characterizes the holistic and transdominial human mind, with perceptions and selfrepresentations of knowledge coming from all specific and interconnected domains (Aureli et al. 2008: 632, 636-637; Bauernfeind et al. 2013: 263-264, 271-273; Foley 2008: 207-210, Mithen 2002: 67-71, 102-111, 139-142; Nordhausen and Oliveira Filho 2015: 36-38). If social consciousness in $P$. troglodytes and, presumably, in the last common ancestor of humans and chimpanzees, is complex enough to fulfill the third of the general fundamentals of ethics according to Ayala, it is something we will not explore here.

We will not go into detail about the ritual of fighting for status among common chimpanzees, but some of its aspects should be highlighted:

1) In the functioning of the status relations, it is normal to recognize that all arrangements are fluid, all coalitions are unstable, and that all rank positions will not be maintained indefinitely, constituting an ethological expectation of 'circulation' in hierarchical positions;

2) When an individual steps up in the status pyramid, another one steps down; this image, coming from the field of primatology, sends us symptomatically to Elias's socio-anthropological perception of power relations in the European 'civilizing process' (Elias 1993) and of Fiori (2014) on the capitalist interstate system:

'The mere preservation of social existence demands a constant expansion in free competition. Whoever does not rise, falls (...). The gain of one in this case is necessarily the loss of another, whether in terms of land, military capacity, money or any other concrete manifestation of social power' (Elias 1993: 134).

3) It is expected that males will confront other males in the clash for ranking positions, considering that rank is associated to reproductive privileges; 
4) It is expected that the struggle for status between individuals will occur through coalitions of agents seeking to support one another, and to intimidate their adversaries;

5) It is expected that a cycle of disputes for status will involve a streak of violations of 'ethical' behavior (refusing to bow down to a higher ranked chimpanzee, denying being touched on the shoulder by a superior, not performing the 'frightened smile' recognized by primatologists); this ethological demonstrations will gradually trigger a status dispute cycle;

6) A dispute can produce coalitions of supporters around the contestants; the victory of a competitor bestows status gains to all supporters, who rise in the social pyramid in detriment of the defeated and his supporters;

7) Disputes are interrupted (until resumed) by something close to a consensus; the members of the group progressively converge to support a particular coalition and its leader, isolating the other competitor and his supporters;

8) The new dominant male takes a conciliatory and pacifying stance;

In this way, by managing a universe of information and choices in a scenario with many moving parts, social intelligence will function as a genuine prosocial ethological brake, which can be subject to anomalous and pathological operations depending on the environmental context. Regarding abnormal conditions generating social pathologies, Stevens speaks of an episode at the London Zoo in 1925, when about a hundred male sacred baboons (Papio hamadryas) were gathered on a small concrete island for exhibition, with six females. Hamadryas baboons, like chimpanzees, have patriarchal societies, although among them, unlike chimpanzees, strict male sexual dominance is practiced, males have uncooperative relations, and form harems (Fleagle 2013: 45-47). The cognitive mechanisms of conflict management in $P$. hamadryas are less complex than in $P$. troglodytes, but they are still in tune with this common trend among the Catarrhini primates (monkeys and great apes of the Old World), that is, to have social intelligence in specialization. In summary, the horde of hamadryas males in the Zoo placed in anomalous 
environmental conditions, engaged in a devastating interpersonal conflict that claimed the lives of forty-two of them, until some sort of dominance hierarchy was established, two years later (Stevens 2002: 265-266).

Thus, in determining the rite and the limits of violence in the conflict between individual agendas, social ethology preserves permanent sociability. It is challenging for us to consider that we, modern humans, often find echoes, images and expectations that, lying deeply hidden in our collective unconscious, project themselves with no warning onto conscious mind, leading us to make choices and influencing our actions as 'voices from the underground' (Jung 2015).

\section{The absence of prosocial ethological restraints in intersocietal relations}

There are no innate prosocial mechanisms in the universe of intersocietal relations among chimpanzees, and we are convinced that they do not exist in modern humans as well. Instances of interaction between distinct social units, when managed exclusively by ethological behavior (as happens with $P$. troglodytes) result in coalitional violence with lethal outcomes. The origins of coalitional intersocietal violence lie in the fragmentation of social groups, precipitated by internal struggles for dominance in a context of environmental pressure or demographic imbalance in the male-female operating ratio (Feldblum et al. 2018: 738). The cycles of internal dispute, in such cases, can result in the split of the social macro-unit; in that case, segmentation of the group into factions during the process leads to secession, denying the homeostatic effects of the 'consensus policy' that normally closes a cycle of status dispute. It follows that distinct foraging areas are constituted, and, in each of these territories a new social unit arises, each one with a hierarchical pyramid of its own. From then on, the contacts between groups will be gradually limited to coalitional lethal violence, in which small groups of males will deliberately invade their neighbor's territory with the aim of eliminating any 'enemy' found astray. This form of 'primitive warfare' is essentially asymmetric, and bears a very interesting resemblance to the practice of ambushes and raids promoted by hunter-gatherer societies, as we can see in the ethnological record. 
An erroneous assessment made by the members of a chimpanzee raiding party, that eventually leads to an encounter with a numerically equivalent (or superior) group of opponents, results in the immediate retreat of the attackers to their home territory. This perspective, of asymmetry, is what qualifies the viability of the coalitional intersocietal violence; lethal aggression by itself is not a rare behavioral phenomenon among mammals, but almost all such events consist of infanticide (on interpersonal level), or of disputes over scarce resources. Interpersonal duels involving reproductive access to females are equally common, although lethality is not the rule. We see, therefore, that the most common contexts of interpersonal lethal aggression derive from a very asymmetrical relationship (infanticide), since adult violence is a high-risk behavior. Symmetry of power can result in the death of any (or both) of the contenders. Thus, the logic of lethality in intersocietal relations would assure itself as standard behavior only if the risks end up controlled somehow. And it is the competence for cooperation and social coordination, highly evolved since the last common ancestor between humans and chimpanzees, which clearly generates an imbalance of power, promoting tactically-explored asymmetry (Ferguson and Beaver 2009; Mithen 2002: 140-141; Wrangham and Peterson, 1996: 5-18, 158-159, 179).

Numbers, like weapons, change the calculation of violence by creating unbalanced power (...). Of course, the same principle guides a wise military leader in our world. Bring massive force to bear. Otherwise, avoid engagement. Isolate and surround, or merely stand and stare. Here is the message taught at Sandhurst at West Point, and no doubt in small village councils throughout the land of the Yanomamö (Wrangham and Peterson 1996: 162).

The balance of power is probably the most effective mechanism for restricting lethal intersocietal violence; once balance of power is questioned by the ethology of male coalitions, aggressors are capable of engaging in lethal violence with minimal risk, and physically eliminate males from outside the social pyramid and from the current patrilineal genetic community. 
A reason for the fragmentation of social units and, as a consequence, for the intersocietal coalitional violence, lies in the processing limitations of the specialized social intelligence, and hence of 'ethics' as behavior. There is a suggested relationship between the encephalization quotient in primates, cerebral neocortex volume, the number of members in a permanent social group and the time spent in socialization. When a growing number of simultaneous relationships have to be analyzed by these dedicated cognitive processes, it can produce a cognitive overflow, hampering the ways of controlling conflict between individual agendas. In these cases of imbalance, the assessment of the status information of every individual in a permanent social group can become more and more sluggish and flawed, so that the effectiveness of the ethological restraints becomes compromised. Information about the social rank of certain individuals can become vague in the mind of an observer, making social strategies compromised by wrong or insufficient assumptions. Permanent factions are formed, and the breakup of the social group in two new units functions as a homeostatic phenomenon, which brings the intensity of social relations to manageable levels. Among modern humans, the metarepresentational cognitive module employs verbal and symbolic language, and material culture, as gigantic buffers of accommodation, expanding the volume of social interactions that can be established simultaneously, providing standardized and instantaneous status information (Aiello and Dunbar 1993), allowing for the wonder of the ultrasociability phenomenon. Thus, although human social cognition also has processing limits, producing pathological behavior when overflowed, the intersocietal lethal conflict between modern humans does not need to result from these same ancestral causes; at an interpersonal level, we know that aggression among hunter-gatherers is a flexible adaptation, susceptible to changes in social and natural environments (Butovskaya 2013), and this is no different for intersocietal violence. What really matters here is how such archaic unconscious complexes can be triggered in the human mind in varied contexts. The condition of 'otherness', the stigma of non-belonging, the hatred towards races, classes or nationalities, all of them resort to deep and unconscious complexes of 'dehumanization', capable of suspending our ethology of conflict regulation and condemn our conspecifics to the same fate as that faced by a chimpanzee slaughtered by an aggressive band. In the words of French and Jack: 
Our troops cannot and should not avoid dehumanizing their enemies to some degree. Just as it is their responsibility to only kill certain people in certain ways at certain times, it is the responsibility of leadership to help them accomplish this by training them to only dehumanize certain people in certain ways at certain times. It takes mental and emotional agility to switch rapidly between different cognitive modes; to go from seeing someone as a 'target to be neutralized' to seeing him as a disarmed and wounded prisoner to whom one must render aid (French and Jack 2015:194).

\section{Metarepresentational transmodularity and the ethics of warfare and peace}

Can we find in chimpanzees and (hypothetically) in our last common ancestor with them an ethology aimed at resolving conflicts between distinct social units? Is there, so to speak, a 'diplomatic cognition' in our evolutionary past, making modern humans and chimpanzees predisposed in normal environmental conditions to resolve conflicts against members of other social groups in order to limit the potential of lethal violence? There are no specialized cognitive modules for this purpose in any of these primate species, nor can chimpanzees employ their social intelligence to mitigate intersocietal conflict. Moreover, the phenomenon that promotes permanent group fission and lethal intersocietal hostility is the exhaustion of specialized social cognition. $H$. sapiens, capable of melting all his innate cognitive domains in a same crucible - the great module of metarepresentation and language -, can see shadows and hear echoes of his deeply unconscious ethology. Because of it, humans are capable not only of employing their social cognition, with forms, functions and algorithms to promote intersocietal violence; they can also employ their cognitive competences to entangle those contents with others of the same ethological nature, and with cultural and specific interpretations of ancestral themes, in order to resignify the 'other', by transmutating him as 'one of us'. After being submitted to the slow process of socialization in a given national culture (normally beginning in childhood), humans are more prone to trust and cooperate with others recognized as members of that culture. This happens because of the expectation of reciprocity that comes from helping and trusting an ingroup member. It is very interesting that in controlled experiments in an online environment, this reputationbased cooperation can be extended to outsiders as well, but with smaller statistical 
significance than that registered in interactions between ingroup members (Romano et al. 2017: 12706). It is possible that markers used as cultural evidence of national outgroup identities are less evident in internet than in face to face interactions, and therefore, the volume of sensorial input available for activating related unconscious complexes is also smaller. Once the 'stranger' is submitted to a 'cognitive alchemy' by the transdominial machinery, he emerges as an 'equal', and therefore subject to the entire framework of ethological instruments of conflict regulation. Nevertheless, neither in chimpanzees nor in modern humans there is any dose of innate capacity that makes us (or them) prone to immediately look at 'the other' as an object of our prosocial restraints; this requires among humans a varied level of conscious and transmodular cognitive effort, that sets in motion behavioral mechanisms originally evolved for another purpose.

\section{Final considerations}

The example of common chimpanzees is very elucidative of the central problem of this work. Even though social life among these primates is ethologically regulated to the point that everyone has the intuitive clarity about what is expected in terms of ethical behavior (an instinctive ethics, according to Ruse and de Waal), this straightforward connection between innate contents and behavior was definitely severed with the Cognitive Revolution, circa 40,000 years in the past, which endowed $H$. sapiens with his metarepresentational mind. Contents and innate forms remain hardwired in the social intelligence of modern humans, immersed in the collective unconscious. The possibilities of a human ethology are not to be mistaken, however; although the mechanisms and the dynamics of the collective unconscious echo in every human action and interaction, individual occurrences of these phenomena are mediated by the holistic consciousness that arises from the module of metarepresentation; this module functions, so to say, like a camara obscura, in which images are projected, coming from the deep recesses of the psyche. In there, these images are cut, mended, combined, and recounted in innovative and creative ways, in narrative and myth. It should not be surprising, however, that the module of metarepresentation is associated with our verbal language, an instrument that makes the social, collective and communicational dimensions of the archetypal images effective. In fact, it is this potential power to share information about 
behavior, trust, cooperation and conflict through verbal language that seems to be at the core of social complexity and human ultrasociability, so that this privileged instrument of transdominiality emerges as an integrating and mediating nexus between ethology and culture. In this way, our 'shared myths' make our sociability possible on a scale beyond family and band levels, producing political units that are, above all, macrofictional narratives, shared through culture - Tribes, States, Empires, Civilizations.

A polity is a sustained community that has structured, sustained relations among its units; over time ever more complex relationships incorporate some earlier ones. With consciousness, or at least with the self-consciousness of social animals, there is a threshold from polity to the political. Human politics has new properties, but it is profoundly rooted in past levels of polity. Human nature evolved from nature (Gustafson 2017: 202).

Unlike lying, an imagined reality is something that everyone believes in, and as long as this communal belief persists, the imagined reality exerts force in the world. No one was lying when, in 2011, the UN demanded that the Libyan government respect the human rights of its citizens, even though the UN, Libya and human rights are all figments of our fertile imaginations (Harari 2015: 40-41).

These narrative fictions work as adaptive 'kludges', allowing us to cope with collective challenges through short-term solutions (in comparison to millions of years sometimes necessary for ethological change to take place through natural selection).

If you tried to bunch together thousands of chimpanzees into Tiananmen Square, Wall Street, the Vatican or the headquarters of the United Nations, the result would be pandemonium. By contrast, Sapiens regularly gather by the thousands in such places. Together, they create orderly patterns - such as trade networks, mass celebrations and political institutions - that they could never have created in isolation. The real difference between us and chimpanzees is the mythical glue that binds together large numbers of individuals, families and groups (Harari 2015: 46). 
In short, if the metarepresentational mind is capable of creatively combining information produced in different durations and cultural contexts, with formulas and contents hardwired in the collective unconscious, and thus, displaying the potential to produce adaptive solutions finely tuned to small changes in the environment:

1) This does not mean that the ability to generate a 'bypass' through 'fast' cultural solutions has simply eliminated the presence of unconscious ethology; on the contrary, it is precisely human culture, with its 'adaptive kludges', that has prevented the pressures of the environment from eliminating non-adaptive behaviors. It is clear that before an 'ecological/ pacifist' ethology ends up selected and hardwired to the collective unconscious (a process that could take millions of years), our shared myths will have been enough to prevent planetary destruction derived from pollution or thermonuclear warfare, or those myths will have provided the degree of cooperation necessary for us to leave behind a dying planet - and carry with us war and devastation to Mars, or to some exoplanet in the Alpha Centauri system. In short, as far as our unconscious ethology is concerned, we will continue insanely devastating our environment and ourselves as we have done since our role in the annihilation of the Eurasian and North American megafauna, and in the first massacres of human history, between 40,000 and 15,000 years ago.

2) This does not also mean that culture overcame natural selection; although this is a notion celebrated by partisans of some kind of 'cultural determinism', it is worth insisting that there is a vast amount of evidence - open to debate, of course - that suggests the persistence in modern humans of cognitive and behavioral elements inherited from our evolutionary past, and generated exactly through selective mechanisms. Thus, it seems inconsistent that natural selection can be admitted as a phenomenon when processes are observable, but not when its results are already consolidated and effective.

3) This does not mean that the metarepresentational mind and that human culture have only generated positive potentials in social terms; let us remember that human ultrasociability was unable to prevent, from the earliest tribes to the contemporary 'planetary society', the schism between the world of those who are close to us and the world of those who are distant in affective, social, psychological and even spatial 
terms. Thus, if ethical behavior (in the sense of Fitzpatrick and Ruse) is mediated by a holistic consciousness in modern humans, this means that this awareness, recurring to deep ingrained ethological algorithms and contents, drives this contents to many innovative directions, but also engulfs them in a tumult of intersections with other cognitive domains, discarding simple and low-energy solutions that could be sufficient for dealing with most of the challenges in a micro-level sociability. And, even if $H$. sapiens was able to automatically activate his innate social ethology and make it work by itself, with no transdominiality and metarepresentation involved, this social ethology would be bound by operational levels restricting permanent interactions to one hundred and a half individuals, approximately, which goes no further than band-level interaction (Aiello and Dunbar 1993: 188-189).

4) This means that our shared myths, and the power of our modern mind and culture, also have limits in the production of ultrasociability. Narrative fictions and images that create the category of insider ('E Pluribus Unum', 'Virtus unita Fortior', 'Einigkeit macht stark') will, by definition, generate outsiders. Ingroup altruism - an important aspect of ultrasociability - could have been enforced in human evolution (genetically or culturally) by a high exposure of social groups to warfare in the Late Pleistocene (Bowles 2009); in view of the fact that intersocietal coalitional violence is inflicted by ingroups against outgroups, it is plausible that this dynamic operates in a feedback loop. In this way, metarepresentational cognition will combine a plethora of cultural contents with elements firmly rooted in social intelligence, allowing unconscious ethological complexes to immerse themselves in these representational contents, making it familiar and credible to each one of us the notion that 'Brazilians' (or 'Finns', or 'Belgians', or 'North-Americans' etc.) are equivalent to 'we', 'a family', 'children of the motherland (or the fatherland)'; in this process, echoes and images hardwired in the social intelligence are invoked by means of metarepresentation, aiming at the production of institutional instruments devoted to the maintenance of what Anderson once called 'imagined communities' (Anderson 2008). Simultaneously, the image of the 'other' is invoked, and once again the specter of the exhaustion of social cognition is conjured up, something that, in times of crisis, facilitates the task of producing and/or endorsing intersocietal coalitional violence, with lethal consequences on a large scale (Steuter and Wills, 2010). 
5) And, finally, it is in the absence of an immediate intuitive ethics that transdominial and metarepresentational cognition, capable of mixing the most disparate elements possible, emerges as a fundamental piece of ethical behavior among modern humans. The capacity for applying ethological complexes to situations originally beyond the scope of primate ethology gives modern humans the possibility of an ethics that can contemplate agents otherwise restricted to a 'dehumanizing' framework (e.g., 'the other', 'the foreigner'). As happens to ultrasociability, an ethics of warfare and peace is also built upon the combination of echoes and images of the collective unconscious, but in this case with a hindrance: the overwhelming torrent of ethological contents invoked in the construction of the ingroup / outgroup dyad, a requirement which, to varying degrees, is needed for the shared myths that inform group identities. That the Peace of Versailles and the rhetoric of 'German humiliation' do not let us forget.

Thus, we can return to the debate on ethical paradigms and formulate the hypothesis of a two-faced ethics. One of the faces is the ancient nature, which looks at what is immediately close and expresses the archaic codes of sociability. The other is our specifically human nature, which is the face capable of seeing what is far (apart from the group and social structures rooted in our genetics) as objects of the same codes, or at least the same principles. The exercise of composing the two faces requires the understanding that all ethical codes are simultaneously an exercise of abstraction and creation from an archaic substrate. Thus, perhaps more than the reflexive rationality proposed by Ayala, which requires a self-critical free will, the foundation of human ethics (especially in its intersocietal sphere) would rely on our social instincts mediated by our outstanding imaginative capacity.

\section{References}

Aiello, L., and Dunbar, R.

1993. Neocortex size, group size, and the evolution of language. Current Anthropology 34: 184-193.

Anderson, B.

2008. Comunidades imaginadas: reflexões sobre a origem e a difusão do nacionalismo. Rio de Janeiro: Companhia das Letras. 
Aureli, F., et al.

2008. Fission-fusion dynamics: new research frameworks. Current Anthropology 49: 627-654.

Ayala, F.

2010. What the biological sciences can and cannot contribute to ethics. In Ayala, F., and Arp, R. (eds.), Contemporary debates in Philosophy of Biology (pp. 14-47). Singapura: Wiley-Blackwell.

Bauernfeind, A., et al.

2013. A volumetric comparison of the insular cortex and its subregions in primates. Journal of Human Evolution 64: 263-279.

Bode, S., et al.

2011. Tracking the unconscious generation of free decisions using ultra-high field fMRI. PLOS ONE 6.

Bowles, S.

2009. Did warfare among ancestral hunter-gatherers affect the evolution of human social behaviors? Science 324: 1293-1298.

Butovskaya, M.

2013. Aggression and conflict resolution among the nomadic Hadza of Tanzania as compared with their pastoralist neighbors. In Fry, D. (ed.), War, peace, and human nature: the convergence of evolutionary and cultural views (pp. 279-296). Oxford: Oxford University Press.

De Waal, F.

2006. The tower of morality. In De Waal, F., Ober, J., and Macedo, S. (eds.), Primates and philosophers (pp. 161-182). Princeton/ Oxford: Princeton University Press.

Elias, $\mathrm{N}$.

1993. O processo civilizador, volume 2: formação do Estado e civilização. Rio de Janeiro: Jorge Zahar Editor.

Feldblum, J., et al.

2018. The timing and causes of a unique chimpanzee community fission preceding Gombe's 'Four-Year War'. American Journal of Physical Anthropology 166: 730-744.

French, S., and Jack, A.

2015. Dehumanizing the enemy: the intersection of neuroethics and military ethics. In Whetham, D., and Strawser, B. (eds.), Responsibilities to protect: perspectives in theory and practice (pp.169-195). Leiden/Boston: Brill/Martinus Nijhoff.

Ferguson, C., and Beaver, K.

2009. Natural born killers: the genetic origins of extreme violence. Aggression and Violent Behavior 14: 286-294. 
Fiori, J. L.

2014. História, Estratégia e Desenvolvimento: para uma geopolítica do capitalismo. São Paulo: Boitempo.

Fitzpatrick, S.

2017. Animal morality: what is the debate about? Biology and philosophy 32: 11511183.

Fleagle, J.

2013. Primate Adaptation and Evolution. San Diego: Elsevier.

Foley, R.

2003. Os humanos antes da humanidade: uma perspectiva evolucionista. São

Paulo: UNESP.

Gustafson, L.

2017. Science, the Deep Past, and the Political. Social Sciences 6: 196-208.

Harari, Y.

2015. Sapiens: uma breve história da humanidade. Porto Alegre: L\&PM.

Jung, C.

2015. O Eu e o Inconsciente. Petrópolis: Vozes.

Keeley, L.

1996. War before civilization: the myth of the peaceful savage. Oxford: Oxford University Press.

Libet, B.

1985. Unconscious cerebral initiative and the role of the conscious will in voluntary action. Behavioral and Brain Sciences 8: 529-566.

Mithen, $\mathrm{S}$.

2002. A pré-história da mente: uma busca das origens da arte, da religião e da ciência. São Paulo: UNESP.

Nordhausen, M., and Oliveira Filho, $\mathrm{P}$.

2015. Nós, primatas. In Neves, W. et al. (eds.), Assim caminhou a humanidade (pp.14-47). São Paulo: Palas Athena.

Romano, A., et al.

2017. Parochial trust and cooperation across 17 societies. PNAS 114: 12702-12707.

Ruse, M.

2010. The biological sciences can act as a ground for ethics. In Ayala, F., and Arp, R. (eds.), Contemporary debates in Philosophy of Biology (pp. 297-315). Singapura: Wiley-Blackwell. 
Soon, C., et al.

2008. Unconscious determinants of free decisions in the human brain. Nature Neuroscience 11: 543-545.

Stevens, A.

2002. Archetype revisited: an updated natural history of the Self. London: Routledge.

Steuter, E., and Wills, D.

2010. 'The vermin have struck again': dehumanizing the enemy in post 9/11 media representations'. Media, War \& Conflict 3: 152-167.

Tooby, J., and Cosmides, L.

2015. The theoretical foundations of evolutionary psychology. In Buss, D. (ed.), The handbook of evolutionary psychology: Volume I. Foundations (pp. 3-87). Hoboken: Wiley and Sons.

Wrangham, R., and Peterson, D.

1996. Demonic males: apes and the origins of human violence. Boston: Mariner. 